\title{
O FUNDO MARINHO DA BAÍA DA ILHA GRANDE, RJ: EVIDÊNCIAS DA AÇÃO DE CORRENTES E DE ONDAS NO CANAL CENTRAL COM BASE EM FORMAS DE FUNDO OBSERVADAS EM REGISTROS DE SONAR (100kHz)
}

\author{
Wellington Ceccopieri Belo \\ Recebido em 13 nov., 2001 / Aceito em 11 ago., 2003 \\ Received Nov. 13, 2001 / Accepted Aug. 11, 2003
}

\begin{abstract}
Based on high-resolution seismic data (sub-bottom profiler 7,0kHz and side scan sonar 100kHz) and geological bottom samples, interesting aspects of the Ilha Grande bay seabed were investigated. The seabed along the eastern-central channel of the bay was characterized by eight bottom sedimentary patterns. Such patterns showed variations in the texture, in the bio-debris content and in the acoustic back-scattering of the sediments. Observed evidences of modern facies transitions from sub-environments dominated by higher energy agents (waves) to facies dominated by lower energy agents (tides, bottom currents) were related to the complex submarine topography and to the coast line orientation. The boundary geometry among the ecofacies also suggests a hydrodynamic control by wave action and bottom currents.

Evidences of these combined effects of waves and currents were approached based on the observed bedforms. Such sedimentary structures are predominantly "mega ripples" located in depths around $16 \mathrm{~m}$, nearby Conceição de Jacareí. There is a textural transition of the bottom sedimentary patterns from westward to eastward. Their crests are NE-SW and ESE-WNW oriented and plenty variable in the form (straight line, wavy, forked, discontinuous). Their heights ranged from 0.2 to $0.55 \mathrm{~m}$ with medium value around $0.35 \mathrm{~m}$. Based on the bedform parameters (wavelength and height) and by using diagrams relating the medium diameter of the grains, to the depth and to the type of the structure, the speed of the flow up to 1,0 meter from the bottom $\left(U_{100}\right)$ was estimated. It varied from $46 \mathrm{~cm} / \mathrm{s}$ (medium sands) to $34 \mathrm{~cm} / \mathrm{s}$ (very fine sands) - from eastward to westward. The medium speed of the stationary flow (U), differently, varied from $54 \mathrm{~cm} / \mathrm{s}$ to $64 \mathrm{~cm} /$ s, respectively. Four studies concerning to wave action indicated that high-energy waves usually observed in the area can contribute to the genesis of the observed bedforms being a good evidence that they could be active. Besides, they could be subject to changes on their geometry along the year. The bottom in this area would be mobile during these events with speed of the oscillatory flows around $50 \mathrm{~cm} / \mathrm{s}$. Upon sporadic events of higher energy $(\sim 80 \mathrm{~cm} / \mathrm{s})$, mainly in the winter, there would be larger readiness of sediment for the bottom transport by currents. Therefore, all these observed characteristics of the eastern-central channel seabed of the bay indicate that waves and currents may act on the sediments sorting by an interplay to the geometry of the bay characterizing different bottom types. The resulting oscillations on the level of energy also can contribute to the bedforms generation, based on sporadic higher energy events.
\end{abstract}

Keywords: high-resolution seismic; ecofacies; bedforms; estimate velocities; Ilha Grande bay.

\section{RESUMO}

Com base em sísmica de alta resolução (perfilador de 7,0kHz e sonar de varredura lateral 100kHz), e amostras de sedimentos, foram investigados aspectos interessantes do fundo marinho na baía da llha Grande. Podem-se evidenciar efeitos combinados de ondas e correntes agindo sobre o fundo marinho, a partir dos índices de ripple (RI) e de paralelismo ( $\mathrm{PI}_{2}$ ) de estruturas sedimentares (formas de fundo) observadas nos registros de sonar. Estruturas estas associadas a padrões sedimentares de fundo (ecofácies). Tais estruturas são predominantemente "mega ripples" localizadas em profundidades em torno de 16m, próximo à Enseada de Conceição de Jacareí onde ocorre uma transição textural de fundo de leste para oeste de um padrão de fundo grosso para um padrão fino. Suas cristas são orientadas a NE-SW e a ESE-WNW e bastante variáveis na forma (retas, onduladas, bifurcadas, descontínuas). Suas alturas variam de 0,2 a 0,55m, com valor médio em torno de $0.35 \mathrm{~m}$. 0 comprimento de onda varia de 10 a $60 \mathrm{~m}$. Velocidades estimadas - unidirecionais para correntes de fundo e orbitais para ação de ondas - com base em parâmetros geológicos das estruturas observadas, sugerem uma possível gradação na magnitude das velocidades $(35-80 \mathrm{~cm} / \mathrm{s})$ na região dos padrões sedimentares de transiç̧ão de fundo. Assim, as feições observadas (faciológicas e morfodinâmicas) do fundo marinho indicam que ondas e correntes ao interagirem com a geometria da baía podem provocar oscilações no nível de energia do meio, atuando no grau de selecionamento dos sedimentos e caracterizando diferentes tipos de fundo.

Palavras-chave. sísmica de alta resolução, ecofácies, formas de fundo, velocidades estimadas, baía da llha Grande.

Centro de Pesquisas Leopoldo A. Miguez de Mello - CENPES - PETROBRAS - Ilha do Fundão, Cidade Universitária, Q7 s/n, 21949-900, 3865-6848 - wceccopieri@petrobras.com.br 


\section{INTRODUÇÃOO}

A baía da llha Grande está localizada entre os meridianos $44^{\circ} \mathrm{W}$ e $44^{\circ} 40^{\prime} \mathrm{W}$ e entre as latitudes $23^{\circ} \mathrm{Se} 23^{\circ} 40^{\prime} \mathrm{S}$, compondo um sistema estuarino com a baía de Sepetiba (Figura 1). Está inserida numa área relativamente bem preservada e é considerada uma região importante onde ocorrem remanescentes da floresta atlântica insular. Possui uma significativa diversidade de ecossistemas marinhos, sendo em grande parte rodeada por costões rochosos, ilhas, praias arenosas e manguezais, características marcantes da costa verde do sudeste Brasileiro, onde a Serra do Mar intercepta o litoral em diversos pontos. Na região do canal central da baía, constata-se que sua geomorfologia é bastante heterogênea. Os processos físicos de circulação e sedimentação atuantes no canal podem apresentar flutuações e resultar em selecionamento dos sedimentos de fundo. Belo e outros (em prep.) investigou e caracterizou a distribuição das ecofácies nesta região. Constatações importantes acerca da ocorrência de diferentes padrões sedimentares de fundo foram abordadas.
0 presente trabalho endereça estas questões integrando informaç̃oes geológicas, geofísicas e oceanográficas na área centro-leste do canal central. Este estudo enfoca interessantes implicações entre as formas de fundo observadas e seus possíveis agentes geradores, considerando 0 tipo de estrutura e 0 padrão sedimentar de fundo (ecofácies) envolvido, e ainda a localização destas estruturas na região.

\section{Formas de fundo}

Formas de fundo são estruturas sedimentares primárias que resultam da ação de ondas e correntes sobre um fundo de sedimentos inconsolidados. A investigação das formas de fundo tem mostrado ser uma importante ferramenta na estimativa e avaliação dos agentes físicos que lhes deram origem. Estudos tanto em laboratório (SOUTHARD, 1975; ALLEN,1982; LEE YOUNG; SLEATH, 1990) quanto no campo (STERNBERG, 1971; AMOS; COLLINS, 1978; ZARILLO, 1982; HENNINGS et al., 2000), vêm sendo realizados no sentido de se estimar a magnitude do escoamento que gera tais estruturas e entender os mecanismos que

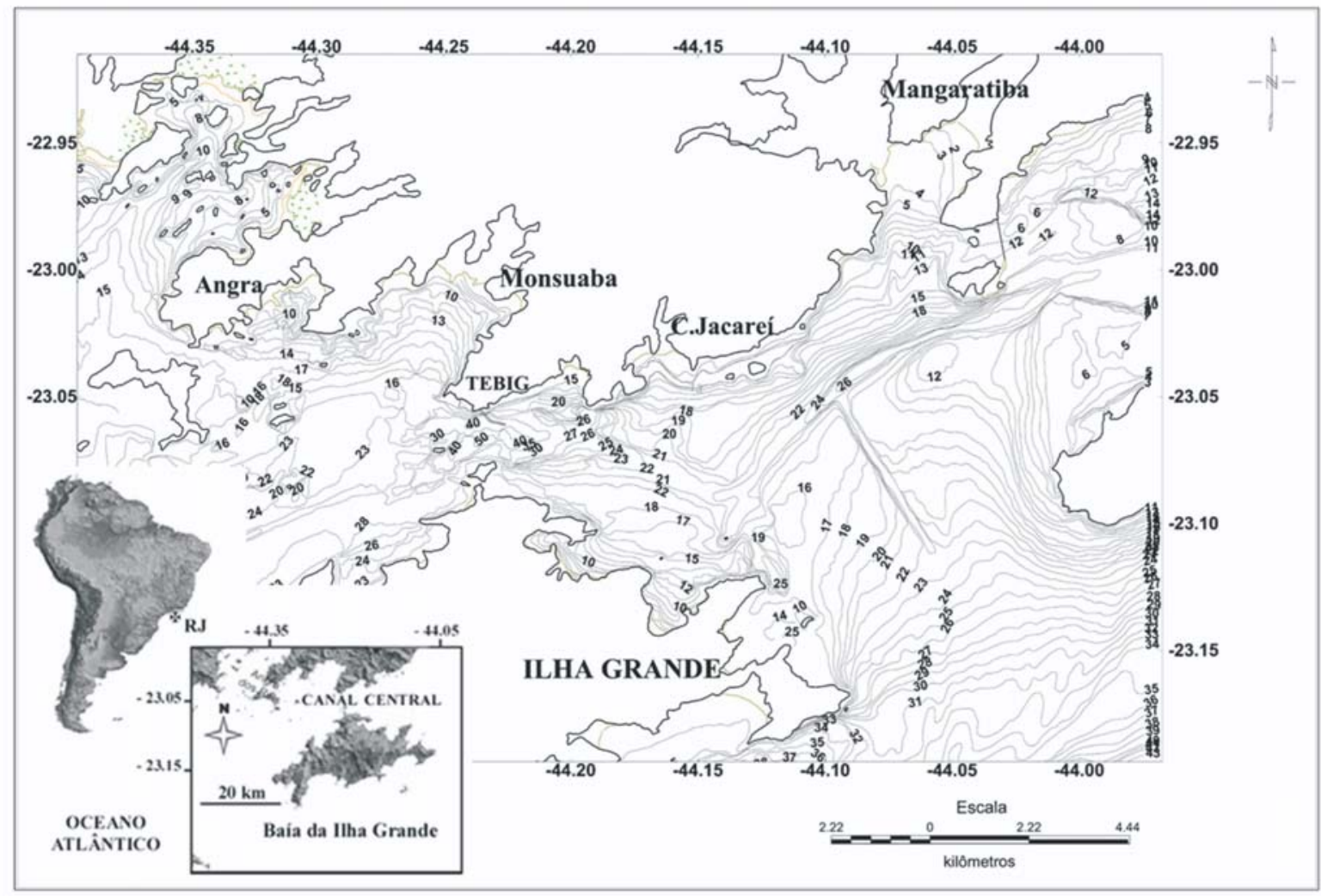

Figura 1 - Área de trabalho. Localização da baía da Ilha Grande e sua batimetria heterogênea. Intervalo de contorno de 1m. Modificado de Dias et. al. (1990). Figure I - Study area. Location of Ilha Grande Bay. Bathymetric contour interval is Im. Modified from Dias et. al (1990). 
as produzem, levando em conta relações entre a energia do agente $\mathrm{e}$ as propriedades do fluido e do sedimento (SOUTHARD, 1975 ; MILLER et al., 1977). Devido à dificuldade de se estabelecer modelos analíiticos acurados que descrevam satisfatoriamente a origem, a morfologia e as condições de escoamento associado a estas estruturas, muitos trabalhos empiricos foram propostos (SOUTHARD, 1971; ALLEN, 1982) e vários diagramas foram elaborados, relacionando a certos tipos de formas de fundo o diâmetro médio dos grãos, 0 estresse cisalhante junto ao leito, a profundidade de ocorrência das estruturas e as velocidades de fluxos tanto unidirecionais - gerados por corrente - quanto oscilatórios gerados por ondas. 0 presente estudo utilizou três desses diagramas com o objetivo de obter estimativas de velocidades de escoamentos de fundo a partir de dados sedimentológicos superficiais, e de parâmetros de formas de fundo, observadas nos registros de sonar de varredura lateral. As características dessas estruturas são então comparadas com dados pretéritos observados de ondas e correntes no canal central.

\section{Ondas}

Estudo realizado por Ferreira da Silva e outros (1996) na porção oeste da baía da Ilha Grande para a companhia FURNAS, na região de Itaorna, mostrou que a média anual das alturas significativas das ondas observadas foi de 1,8m e seu período de 10,3s, sendo estes valores máximos no inverno (junho $\mathrm{Hs}=2,42 \mathrm{~m} ; \mathrm{Tc}=11,9 \mathrm{~s}$ ) e mínimos no verão (dezembro $\mathrm{Hs}=1,3 \mathrm{~m} ; \mathrm{Tc}=9,7 \mathrm{~s}$ ). Seus resultados mostram a distribuição de ocorrência de ondas nesta região da baía. As ondas mais freqüentes durante 0 ano na porção oeste provêm da direção $\mathrm{E}(24 \%)$, e da direção NE (16\%), seguidas das ondas de SE (10\%), S (11\%) e SW (12\%). As ondas de SE, Se SW foram mais freqüentes na primavera $(12,14$ e $15 \%$, respectivamente). As alturas significativas mais freqüentes ao longo do ano (80\%) estão entre 0,3 e 1,5m, sendo 0 verão a estação com menores alturas de onda $(0,3$ a $0,5 \mathrm{~m})$ com $54 \%$ das observaçõos nesse período. № inverno, esta variação passa a 38,5\%. As ondas de maiores alturas significativas $(1,6$ a 3,5m) representam $12 \%$ das observações, exceto no inverno quando chegam a $14,5 \%$. Destas, $3 \%$ das observações estão na faixa de 2,6 a 3,5m, exceto no inverno com $6 \%$ de ocorrência. Estas alturas estão mais vinculadas a ondas de direções E, SE e SW. A configuração geográfica da linha de costa na regic̃o da baía da llha Grande facilitaria a penetração das ondas do quadrante $S-S E$, que teriam maior ação na região de Mambucaba (na porção oeste) e em todo litoral a oeste da Ilha Guaíba (na porção leste), região de Conceição de Jacaré, enquanto as oriundas de outros quadrantes seriam barradas ou parcialmente barradas, como as de SW.

\section{Correntes}

Com base em estudos de vários autores (CORRÊA et al., 1997; IKEDA et al., 1989; SIGNORINI, 1980; FRAGOSO, 1999), pode-se inferir que os processos de transporte atuantes na parte central da baía da llha Grande seriam predominantemente de baixa energia e a sedimentação controlada por processos hidrodinâmicos. De acordo com Ikeda e outros (1989) e Signorini (1980), a baía da Ilha Grande teria uma circulação horária quase estacionária, independente das oscilaçōes de maré e de vento, sugerindo, porém, que parte dessa circulação seja intensificada por este último. Signorini (1980) propôs que o mecanismo de circulação seria similar ao de um sistema estuarino composto pelas baías de llha Grande e de Sepetiba, parcialmente misturado e possuindo componentes de circulação gravitacional, residual de maré e gerada pelo vento. Sua circulação seria definida por um fluxo quase estacionário para leste induzido por gradientes de densidade. Nesses estudos, os campos de densidade e de velocidade de correntes indicaram oscilações (seiches), devido à geometria local e à estratificação da coluna d'água, associadas à descarga da baía de Sepetiba e à penetração sazonal da ACAS (Água Central do Atlântico Sul) (MIRANDA et al.,1977; IKEDA; STEVENSON, 1982).

Medições de corrente realizadas no pier do TEBIG (Terminal da Petrobras) no canal central em 1998 por B. Kjerfve e G. T. M. Dias (Figura 2) mostraram que estas apresentam reversões esporádicas a $15 \mathrm{~m}$ de profundidade, porém, com um escoamento predominante para leste. Pode-se observar com base nestas medições, a possível influência de forçantes atmostéricos no comportamento das correntes, evidenciados por eventos de maior velocidade das mesmas, da ordem de $60 \mathrm{~cm} / \mathrm{s}$ para leste, associados a componentes de períodos superiores a 30 horas. Segundo Fragoso (1999), isto pode ser observado pelo pico de período entre 2 e 5 dias no seu espectro de energia. Houve também períodos em que 0 escoamento foi predominante para oeste com velocidades de pico da ordem de $30 \mathrm{~cm} / \mathrm{s}$. Estas medições, segundo Fragoso (1999), mostraram ainda que 0 período natural de oscilação do sistema llha Grande/Sepetiba é bastante próximo a 6 horas.

\section{MATERIAL \& MÉTODOS}

Foi utilizado o seguinte conjunto de dados na realização deste trabalho (Figura 3):

- Dados sedimentológicos do Mapa Geológico/Geomorfológico da baía da llha Grande (DIAS et al., 1990), na escala 1:80000, com base nas amostras superficiais de sedimentos existentes de campanhas pretéritas; 
- Dados batimétricos analógicos também do Mapa Geológico/ Geomorfológico da baía da llha Grande (DIAS et al., 1990), na escala 1:80000, com intervalo de contorno de 1,0m;

- Dados geofísicos de sísmica rasa (ecobatimetria, perfilador de 3,5$7,0 \mathrm{kHz}$ e sonar de varredura lateral $100 \mathrm{kHz}$ ) obtidos em campanhas de campo;

- Dados sedimentológicos superficiais de fundo coletados em campanha de campo, em locais espećíicos a fim de se confirmar os padrões geofísicos do eco.

\section{Dados geológicos}

Os dados geológicos pretéritos são provenientes de 153 amostras sedimentológicas de Mahiques (1987), classificados de acordo com Dias (1996), e um mapa de Dias e outros (1990) que mostra prováveis transições texturais. Esses mapas foram utilizados no planejamento das linhas geofísicas.

Foram coletadas adicionalmente amostras sedimentares de verificação em alguns locais onde se julgou necessário maior definição

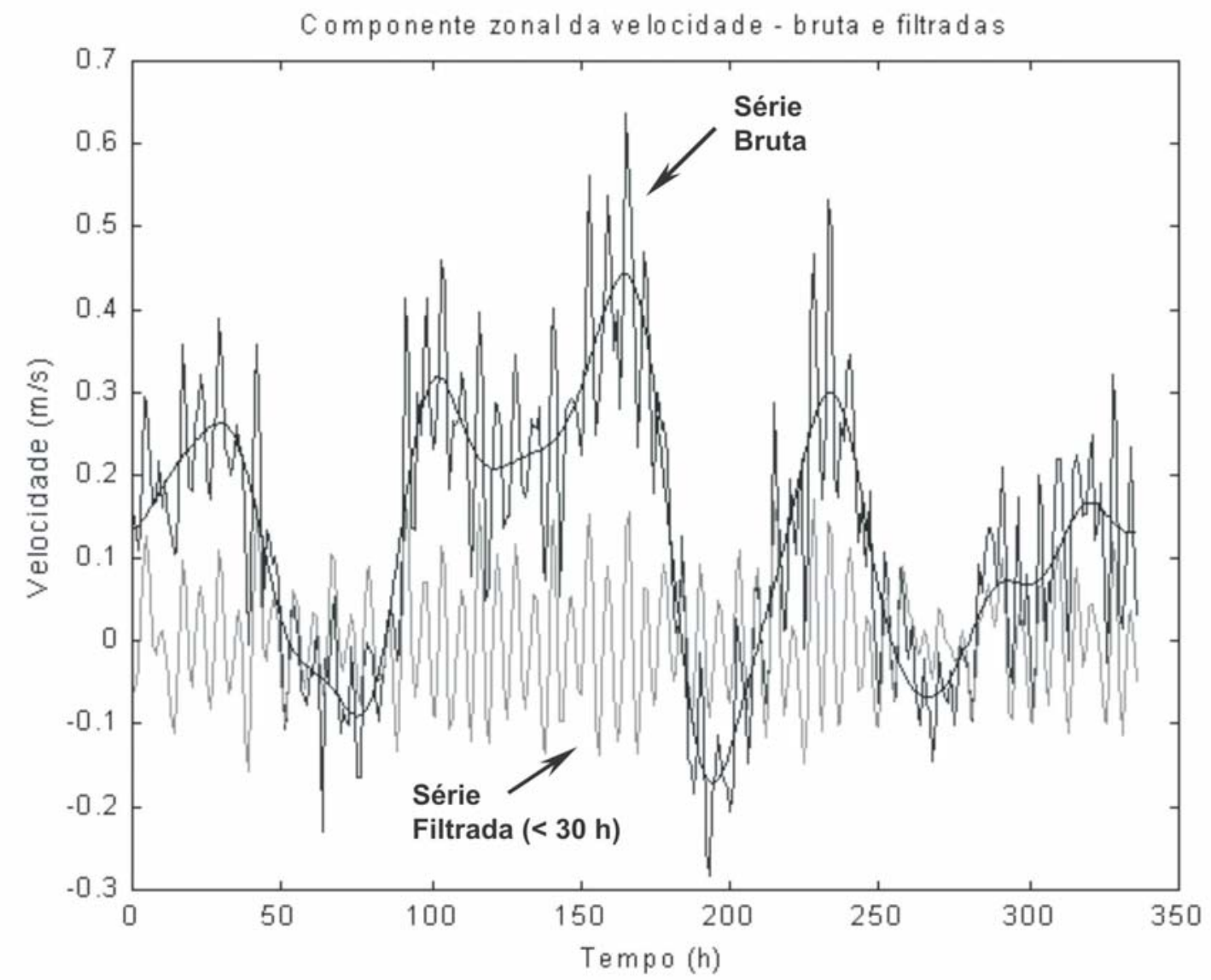

Figura 2 - Séries temporais de correntes medidas no canal central durante 14 dias, a 15m de profundidade, em frente ao TEBIG por B. Kjerfve e G. T. M. Dias em 1998. Em cinza escuro está a série bruta. Em cinza claro a série filtrada, considerando apenas as componentes com período de maré (abaixo de 30 horas). Em preto está a componente com período maior do que 30 horas, indicando uma possível influência de forçantes atmosféricas na circulação da região do canal central. Modificado de Fragoso (1999).

Figure 2 - Time-series of currents in the central channel. Data were collected on an interval of 14 days, at $15 \mathrm{~m}$ depth. Current meter was located off the Petrobras Oil Terminal (TEBIG). Data collected by B. Kierfve and G.T.M. Dias in 1998 and interpreted by Fragoso (1999). Dark gray shows the raw data. Light gray shows the filtered data, considering only the tidal components lower than 30 hours. In black are the components greater than 30 hours, showing a possible influence of the atmospheric forces in the central channel hydrographic circulation. 


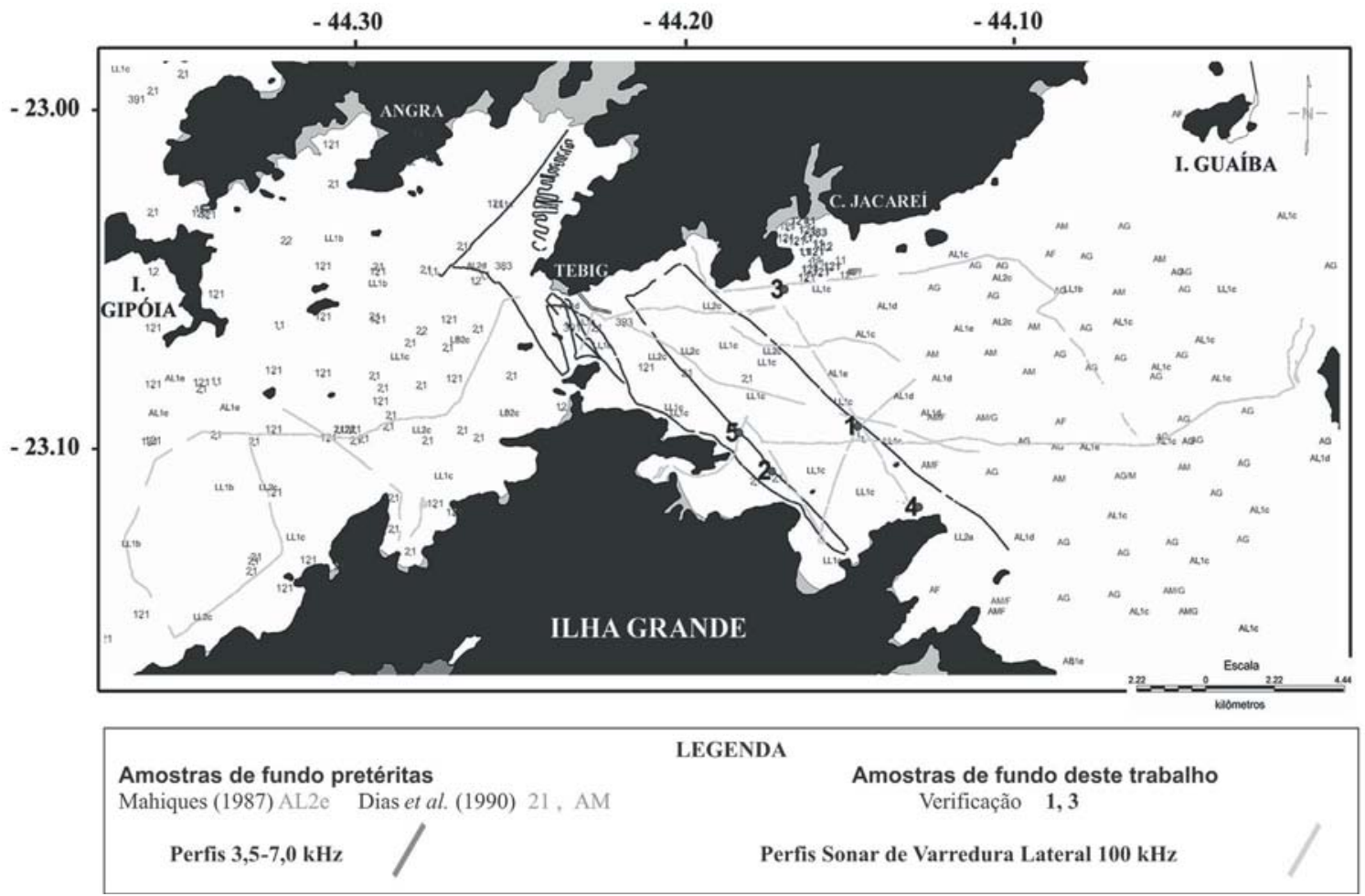

Figura 3 - Mapa base de dados geofísicos e geológicos. Figure 3 - Location of geological (bottom-sediment superficial samples) and geophysical data (3.5 and $7 \mathrm{kHz}$ sub-bottom echo-sounder and side scan sonar, 100 kHz, images).

geológica, confrontando-as com as amostras pretéritas. Estas amostras de verificação foram coletadas com um amostrador tipo van-Veen sobre as linhas de levantamento geofísico do sonar e do perfilador, onde seus traçados se cruzaram. A análise granulométrica foi realizada através de peneiramento da fração grossa e pipetagem da fração fina de acordo com Suguio (1973), em duplicata, com e sem eliminação do carbonato de cálcio com ácido clorídrico (30\%) presente nas amostras, a fim de se verificar onde a fração biodetrítica carbonática seria mais importante. 0 teor de matéria orgânica foi obtido com base no uso de peróxido de hidrogênio (10\%). A classificação comparativa foi feita de acordo com Shepard \& Moore (1954) e Larsonneur e outros (1982).

\section{Dados geofísicos}

Os dados geofísicos foram obtidos por ecossondagens contínuas, posicionados com DGPS. Foram 156,32km de linhas de imageamento do fundo submarino com sonar de varredura lateral (EG \& $G-260$, $100 \mathrm{kHz}$ ), e 78,67km em perfilagens de subfundo (Raytheon RTT 1000 A - PTR 7,0kHz) nos primeiros 10-15m de camada sedimentar, realizados em três campanhas (maio e dezembro de 1999 e setembro de 2000). Com base nos registros geofísicos, foram identificados os tipos de ecocaráter superficial observados com o perfilador e os padrões de reflexão do sonar. Os dados geofísicos são correlacionados nos pontos onde os respectivos traçados se cruzam (Figura 3), e ainda com as informações de amostras dos sedimentos superficiais neste ponto de cruzamento. A combinação dessas informações permitiu a caracterização dos padrões sedimentares de fundo (ecofácies) por Belo et al. (em prep.) (Figura 4).

\section{Classificação das formas de fundo}

As formas de fundo observadas foram classificadas (REINECK; SINGH, 1975) utilizando relações entre índices obtidos a partir do comprimento de onda (L), da altura $(H)$ e das projeções das faces das estruturas no fundo marinho. Com base nesses parâmetros, foram obtidos 0 índice de ripple (RI) e o índice de paralelismo $\left(\mathrm{PI}_{2}\right)$, usados como critérios na classificação segundo 0 agente gerador principal das formas de fundo. Outros índices não puderam ser usados pela impossibilidade de se estimar os parâmetros necessários nos registros de sonar. Para interpretar as estruturas e estimar os parâmetros ( $(\mathrm{L}$ e H) utilizados nos 


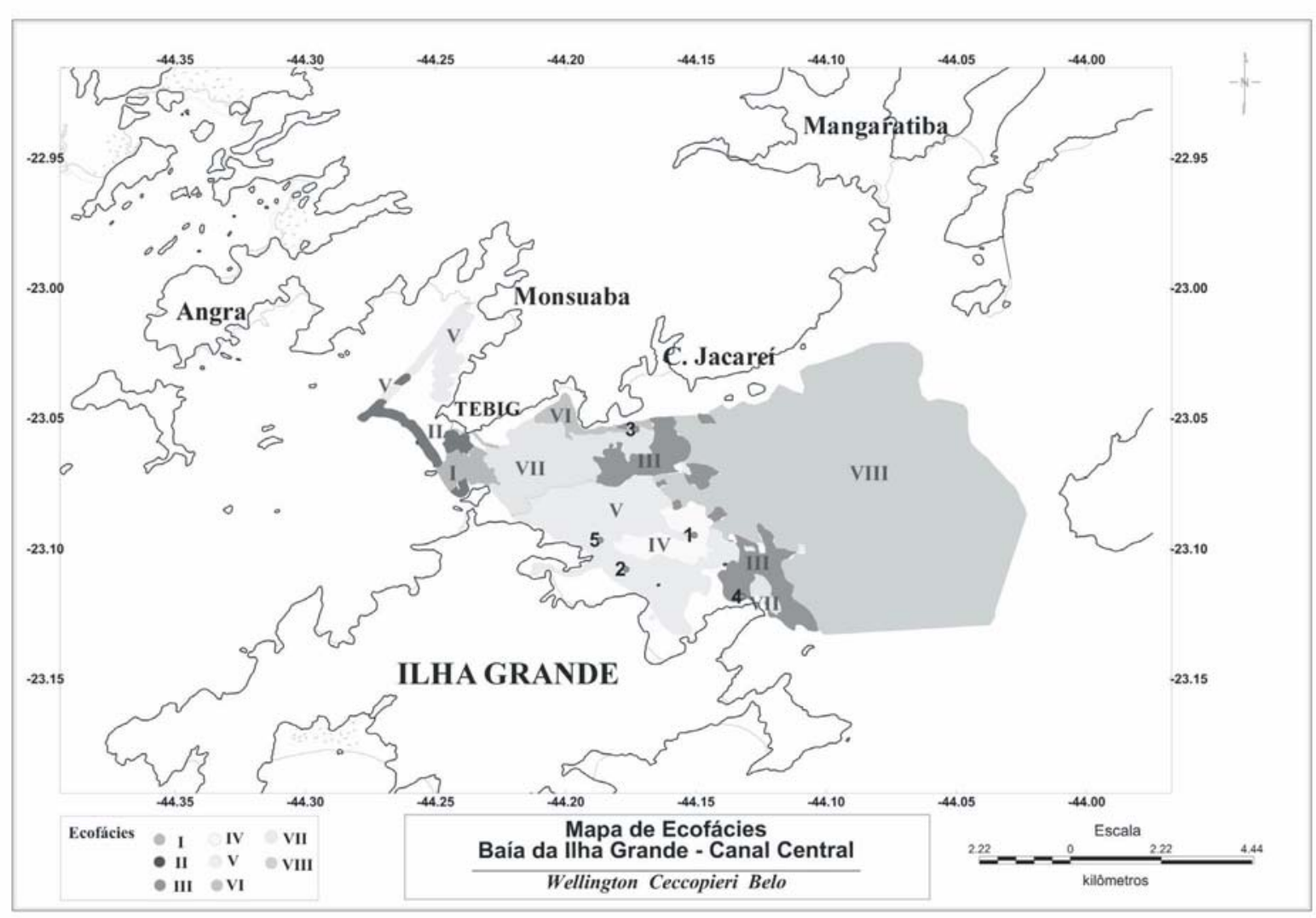

Figura 4 - Ecofácies da porção centro-leste do canal central da baía da Ilha Grande: (I) - Lama terrígena; (II) - Areia litobioclástica média; (III) - Areia litobioclástica muito fina; (IV) - Lama terrígena arenosa; (V) - Lama terrígena; (VI) - Marga arenosa; (VII) - Marga arenosa; (VIII) - Areia litoclástica média a grossa.

Figure 4 - Echo-facies distribution on the central-eastern portion of the Ilha Grande Bay central channel. (I) Terrigenous mud; (VI) Sandy maerl; (VII) Sandy maerl; (VIII) Coarse to medium litho-clastic sand.

cálculos dos índices, utilizou-se o método proposto (FISH; CARR, 1990), através da semelhança de triângulos, e do reconhecimento de áreas de forte reflexão e de zonas de sombra. A faixa de variação dos índices utilizados, em função do principal agente, é apresentada na Figura 5.

\section{Velocidades estimadas}

Devido às limitações inerentes a esta abordagem, 0 presente estudo não teve a intenção de definir números conclusivos. Mas, com base nas estruturas sedimentares observadas e em suas características, ter uma noção da ordem de grandeza dos agentes envolvidos na sua geração. Assim, com base nos parâmetros das formas de fundo, e nas ecofácies onde foram observadas estas estruturas, foram utilizados três diagramas da literatura que exprimem relações empíricas entre algumas variáveis para: a) estimar a velocidade de cisalhamento média do fluxo a 1,0m do fundo $\left(U_{100}\right)$ em função do diâmetro médio do sedimento (MILLER et al., 1977) (Figura 6); b) estimar a velocidade média do fluxo $(U)$ em função do tipo de estrutura sedimentar presente a uma dada profundidade e do diâmetro médio do sedimento (RUBIN; MCCULLOCH, 1979); e c) estimar a velocidade orbital limite (Uml), do fluxo oscilatório que uma certa onda imprime ao fundo marinho, sendo capaz de erodi-lo, em função do diâmetro médio do sedimento e do período desta onda (KOMAR; MILLER, 1975), no caso uma onda com $\mathrm{T}=10 \mathrm{~s}$ (Figura 7). Velocidades orbitais de onda (Um2) foram obtidas também para quatro condições observadas por Ferreira da Silva et al., (1996), com base em Wigel (1954): média das alturas significativas máximas de verão, média das alturas significativas máximas anuais, média das alturas significativas máximas de inverno, média das alturas significativas máximas em eventos esporádicos. Estes foram comparados à velocidade orbital limite (Uml). 


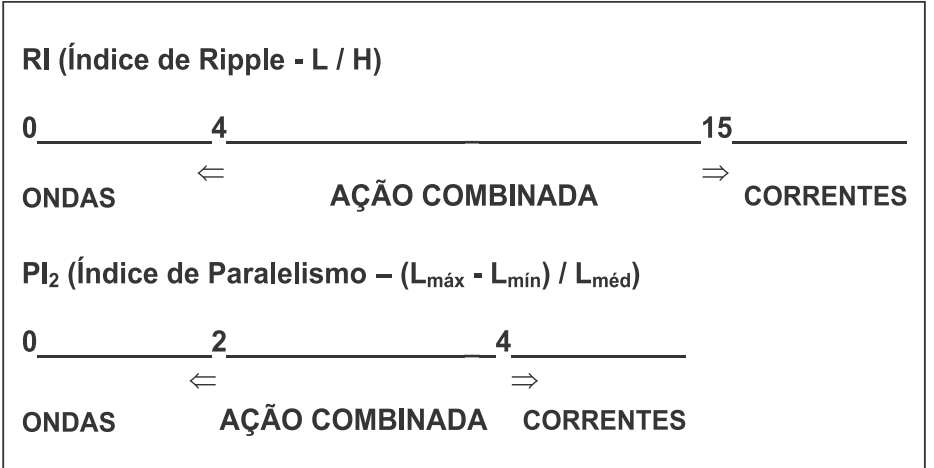

Figura 5 - Escala mostrando a faixa de variação dos índices RI (índice de ripple) e $\mathrm{PI}_{2}$ (índice de paralelismo), segundo a ação de ondas e correntes, com base na classificação de Reineck \& Singh (1975).

Figure 5 - Variation intervals of the Ripple index (RI) and Parallel Index (PI)) according to the action of waves and currents, based on the classification of Reineck \& Singh (1975).

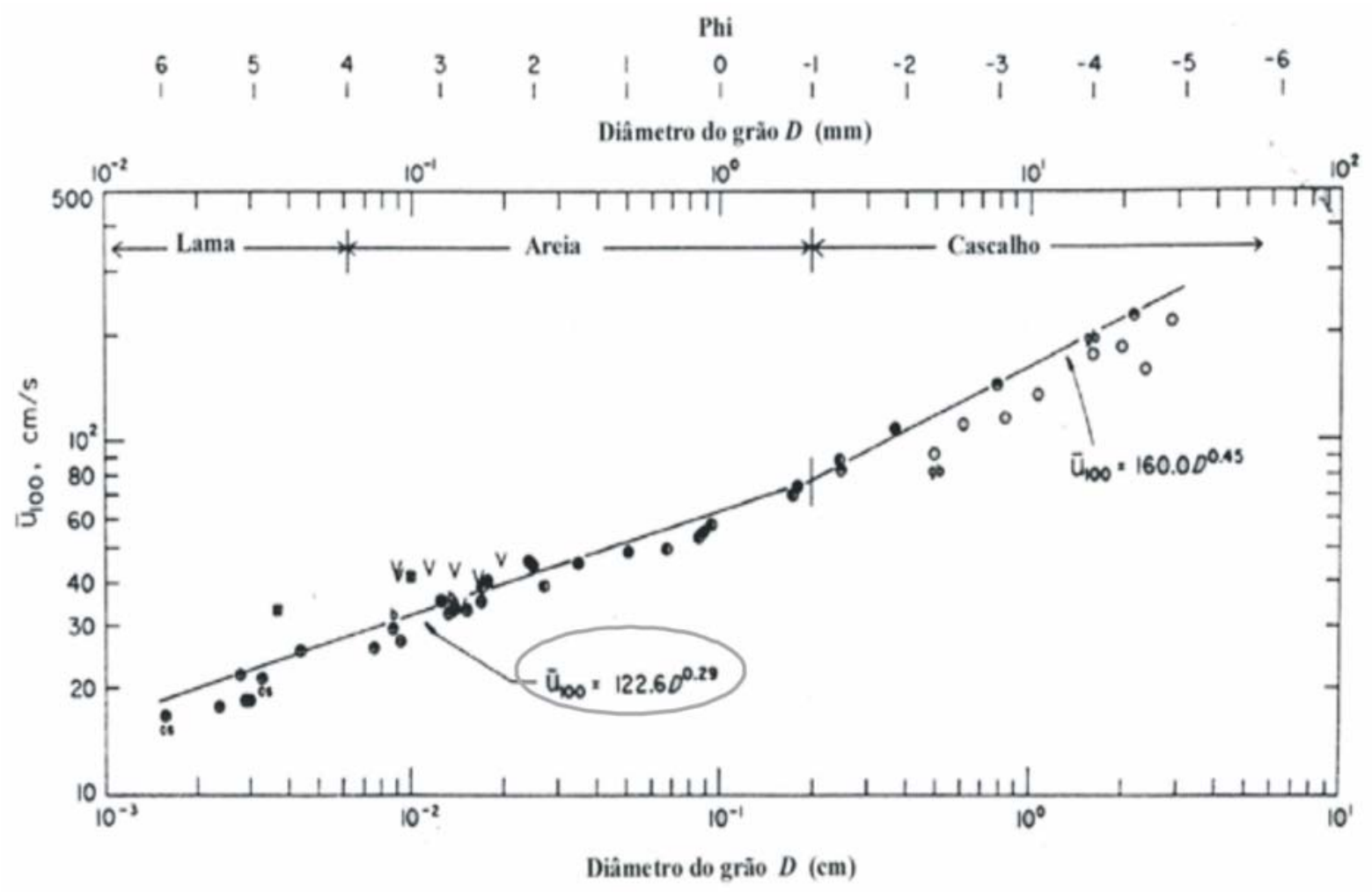

Figura 6 - Equações empíricas no cálculo da velocidade média de cisalhamento do fluxo a $100 \mathrm{~cm}$ do fundo $\left(U_{100}\right)$ em função do diâmetro médio dos grãos (em centímetros). Equação utilizada marcada com a elipse. Adaptado de Miller et. al. (1977). Figure 6 - Empirical equations used to calculate the medium shear velocity of the flow at $100 \mathrm{~cm}$ from the bottom $\left(U_{100}\right)$ as a function of the medium grain size (in cm). The used equation is shown inside the ellipse on the graph. Adapted after Miller et al (1977). 


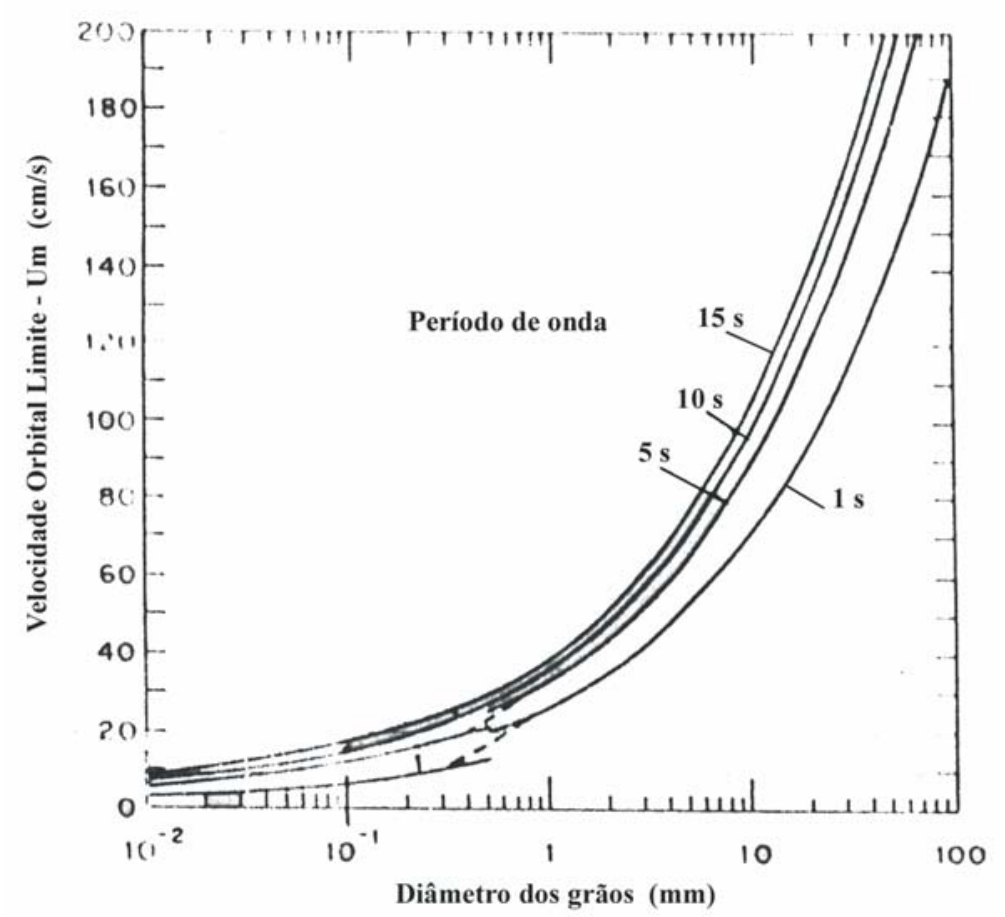

Figura 7 - Velocidade orbital máxima próxima ao fundo (Um) para o limite de movimento dos grãos sob ondas de diferentes períodos. Neste estudo, o período utilizado foi de 10s. Adaptado de Komar \& Miller (1975).

Figure 7 - Maximum orbital velocity near the bottom $\left(U_{n}\right)$ at the grain movement threshold for different wave periods. In this study the used period was 10 s. Adapted after Komar \& Miller (1975).

\section{RESULTADOS E DISCUSSÃO}

Neste trabalho, dados geofísicos, oceanográficos e dados sedimentológicos foram analisados de forma integrada, na qual foram observadas formas de fundo associadas a alguns padrões sedimentares superficiais de fundo (ecofácies). A seguir, evidências da atuação de forçantes oceanográficos na distribuição da ocorrência destes padrões serão abordadas.

\section{Parâmetros das formas de fundo}

As "mega ripples" são dominantes na região centro-leste da baía da Itha Grande, principalmente ao largo da Enseada de Conceição de Jacareí, em profundidades em torno de 15 a 17m (Figura 8) onde há uma transição de oeste para leste de ecofácies (padrões sedimentares finos para grossos) (Tabela 1). Suas cristas são orientadas a NE-SW e a ESE-WNW e bastante variáveis na forma (retas, onduladas, bifurcadas e descontínuas). Suas alturas variam de 0,2 a $0,55 \mathrm{~m}$, com valor médio em torno de 0,35m. 0 comprimento de onda varia de 10 a 60m. Entre a llha Grande e a Restinga de Marambaia, uma extensa região de areias médias a grossas é marcada por pequenas ondulações orientadas SSENNW com alturas menores que $20 \mathrm{~cm}$ e cristas onduladas regularmente espaçadas de $8,6 \mathrm{~m}$ em torno de $21 \mathrm{~m}$ de profundidade. Várias marcas aleatórias de orientações diversas também foram observadas.

A análise dos índices $\mathrm{RI}$ e de $\mathrm{Pl}_{2}$ sugere que existam efeitos combinados de correntes e ondas na maioria dos locais onde as formas de fundo foram observadas. Os valores de RI mostraram a predominância de correntes agindo sobre o fundo, havendo uma diminuição do efeito das mesmas de leste para oeste. Por outro lado, os valores de $\mathrm{Pl}_{2}$ indicam influência relativamente maior de ondas a oeste, na direção do TEBIG.

As velocidades estimadas, com base nos parâmetros geológicos das ecofácies e das formas de fundo (Figura 9), nos dão uma certa noção da magnitude de diferentes fluxos (unidirecionais e oscilatórios) atuando sobre o fundo marinho. Os resultados obtidos podem ser observados na Tabela 2. Observa-se que a ordem de grandeza das velocidades estimadas é consistente com as velocidades observadas no canal central na Figura 2. É importante, porém, ressaltar que as considerações feitas aplicando os diagramas excluem a influência da 


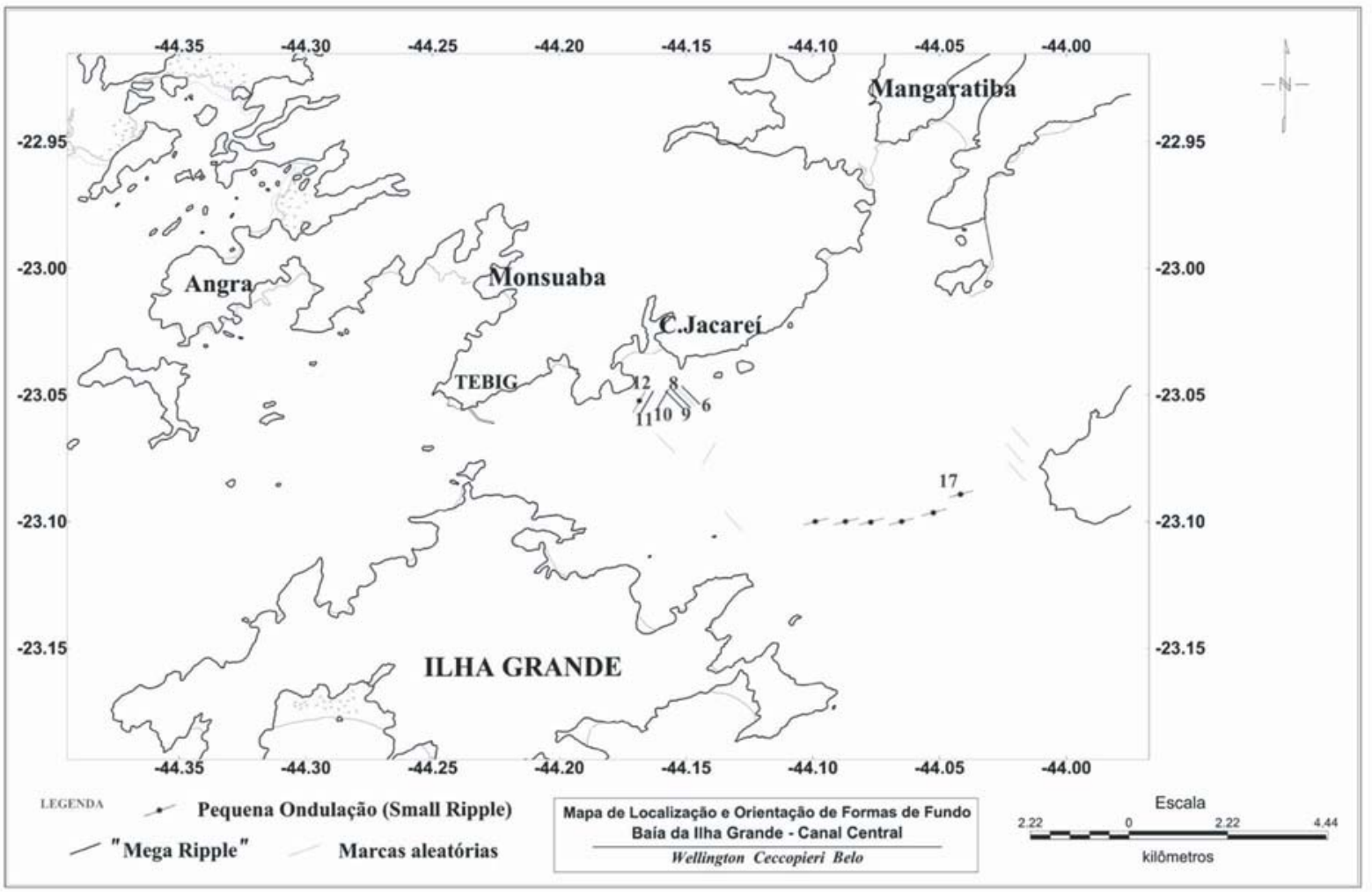

Figura 8 - Localização e orientação das estruturas sedimentares, a partir de registros de sonar de varredura lateral (100kHz). Figure 8 - Location and orientation of the sedimentary bedforms observed in side scan sonar (100 kHz) images.

Tabela 1 - Características das formas de fundo observadas com sonar de varredura lateral (100kHz) nas campanhas de maio de 19991 e setembro de 2000², na região centro-leste do canal central da baía da Ilha Grande. Classificação segundo Reineck \& Singh (1975).

Table 1 - Characteristics of the bedforms observed in side scan sonar images surveyed on may, 1999 and September 2002, in the central-eastern portion of the Ilha Grande Bay central channel. Classification after Reineck \& Singh (1975).

\begin{tabular}{|c|c|c|c|c|c|c|c|c|c|}
\hline Local & $\begin{array}{l}\mathrm{L} \\
\text { (m) }\end{array}$ & $\begin{array}{c}H \\
(m)\end{array}$ & Orientação & $\begin{array}{l}\text { Prof. } \\
\text { (m) }\end{array}$ & Crista & $\begin{array}{l}\text { Diam } \\
(\mathrm{mm})\end{array}$ & $\mathrm{RI}$ & $\mathrm{PI}_{2}$ & Classificação \\
\hline 6 & $\begin{array}{c}45 \\
40-50\end{array}$ & 0.30 & NE-SW & 16.0 & $\begin{array}{c}\text { Reta } \\
\text { Contínua }\end{array}$ & $\begin{array}{l}0.354 \\
\text { (AM) }\end{array}$ & 112.5 & 0.11 & Mega Ripple' \\
\hline 8 & $\begin{array}{c}41 \\
20-62\end{array}$ & 0.55 & NE-SW & 16.0 & $\begin{array}{l}\text { Reta } \\
\text { Contínua }\end{array}$ & $\begin{array}{c}0.176 \\
\text { (AF) }\end{array}$ & 74.50 & 1.02 & Mega Ripple' \\
\hline 9 & $\begin{array}{c}20 \\
10-30\end{array}$ & 0.45 & NE-SW & 16.3 & $\begin{array}{c}\text { Reta } \\
\text { Descontínua }\end{array}$ & $\begin{array}{c}0.176 \\
\text { (AF) }\end{array}$ & 44.40 & 1.00 & Mega Ripple' \\
\hline 10 & $\begin{array}{c}11.5 \\
10-13\end{array}$ & 0.40 & ESE-WNW & 16.3 & $\begin{array}{l}\text { Ondulada } \\
\text { Bifurcada }\end{array}$ & $\begin{array}{c}0.176 \\
\text { (AF) }\end{array}$ & 28.75 & 0.26 & Mega Ripple’ \\
\hline 11 & $\begin{array}{c}12.5 \\
10-15\end{array}$ & 0.30 & ESE-WNW & 16.5 & $\begin{array}{l}\text { Ondulada } \\
\text { Contínua }\end{array}$ & $\begin{array}{c}0.176 \\
\text { (AF) }\end{array}$ & 41.67 & 0.40 & Mega Ripple' \\
\hline 12 & 5.0 & 0.25 & ESE-WNW & 16.5 & $\begin{array}{l}\text { Ondulada } \\
\text { Contínua }\end{array}$ & $\begin{array}{l}0.125 \\
\text { (AMF) }\end{array}$ & 20.00 & N/D & Pequena Ondulaçãa ${ }^{1}$ \\
\hline 17 & 8.6 & $>0.20$ & SSE-NNW & 21.0 & $\begin{array}{l}\text { Ondulada } \\
\text { Contínua }\end{array}$ & $\begin{array}{l}0.707 \\
(A G)\end{array}$ & 43.10 & $\mathrm{~N} / \mathrm{D}$ & Pequena Ondulaçãa ${ }^{2}$ \\
\hline
\end{tabular}




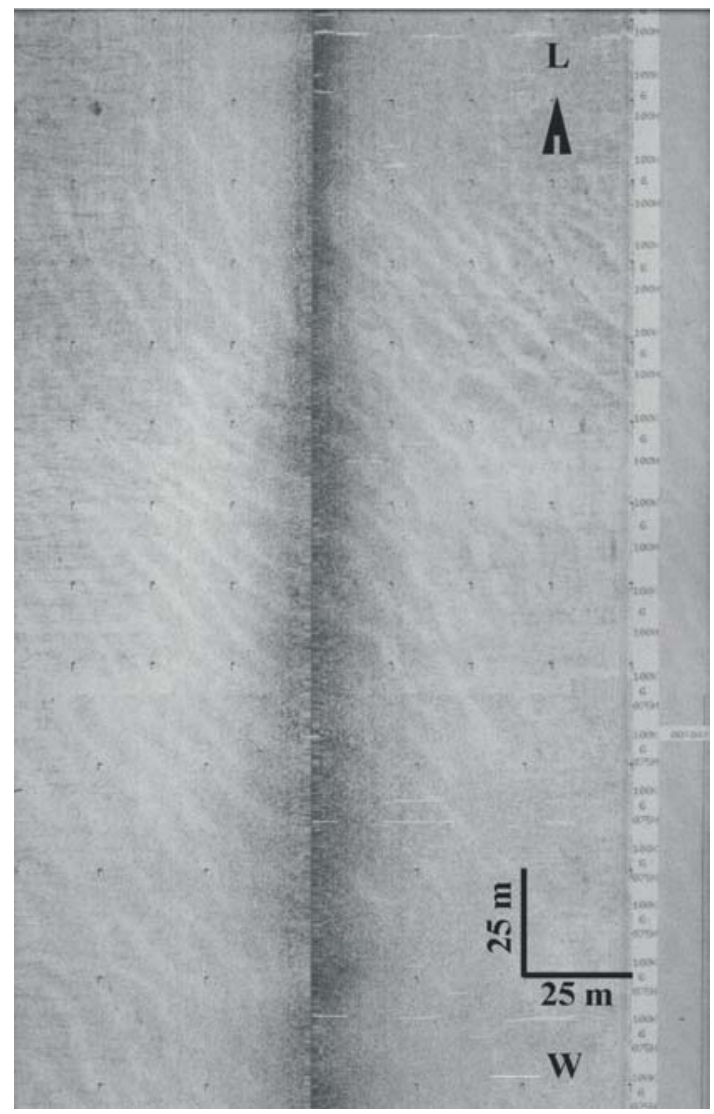

\author{
10 \\ "Mega Ripple" \\ $\lambda_{\text {médio }}=11.5 \mathrm{~m}$ \\ $\lambda_{\text {min }}=10 \lambda_{\text {max }}=13 \mathrm{~m}$ \\ $\mathrm{H} \Rightarrow 0.40 \mathrm{~m}$ \\ Orientação $\Rightarrow$ ESE-WNW \\ Profundidade $\Rightarrow 16.3 \mathrm{~m}$ \\ Cristas $\Rightarrow$ Onduladas, contínuas com poucas bifurcações

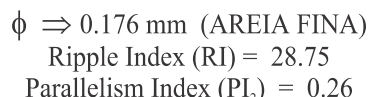 \\ Parallelism Index $\left(\mathrm{PI}_{2}\right)=0.26$ \\ Provável origem : Ondas e Correntes (transversos)
}

Figura 9 - Exemplo de esquema de características de estruturas sedimentares observadas com base em registros de sonar de varredura lateral (100kHz).

Figure 9 - Example of the bedforms observed on side scan sonar (100 kHz) image.

Tabela 2 - Estimativas de diferentes velocidades de fluxos próximo ao fundo associados às formas de fundo observadas com sonar de varredura lateral, com base em: (a) - Miller et al. (1977); (b) - Rubin \& McCulloch, (1979); e (c) - Komar \& Miller (1975).

Table 2 - Estimated flow velocities near the bottom in association with the bedforms observed on side scan sonar images, based in: (a) - Miller et al. (1977); (b) - Rubin \& Mcculloch (1979) and (c) Komar \& Miller (1975).

\begin{tabular}{|c|c|c|c|c|c|c|}
\hline & Local & $\begin{array}{l}U_{100}(\mathrm{a}) \\
\left(\mathrm{cm} \cdot \mathrm{s}^{-1}\right)\end{array}$ & $\begin{array}{c}\text { U.(b) } \\
\left(\mathrm{cm} . \mathrm{s}^{-1}\right)\end{array}$ & $\begin{array}{c}U_{*}(\mathrm{a}) \\
\left(\mathrm{cm} \cdot \mathrm{s}^{-1}\right)\end{array}$ & $\begin{array}{c}U:(b) \\
\left(\mathrm{cm} \cdot \mathrm{s}^{-1}\right)\end{array}$ & $\begin{array}{l}U_{\mathrm{ml}}(\mathrm{c}) \\
\left(\mathrm{cm} \cdot \mathrm{s}^{-1}\right)\end{array}$ \\
\hline & 6 & 46.52 & 54.0 & 0.37 & 0.40 & 28.0 \\
\hline & 8 & 37.99 & 62.0 & 0.38 & 0.43 & 18.0 \\
\hline & 9 & 37.99 & 63.5 & 0.38 & 0.43 & 18.0 \\
\hline & 10 & 37.99 & 63.5 & 0.38 & 0.43 & 18.0 \\
\hline & 11 & 37.99 & 64.5 & 0.38 & 0.44 & 18.0 \\
\hline & 12 & 34.40 & 64.5 & 0.32 & 0.44 & 16.0 \\
\hline & 17 & 56.86 & 56.0 & 0.41 & 0.41 & 35.0 \\
\hline OBS: & $\begin{array}{l}U_{100} \\
U . \\
U . \\
U_{m 1}\end{array}$ & $\begin{array}{l}\text { velocidade de cisalhamento mé } \\
\text { velocidade média do fluxo estac } \\
\text { velocidade unidirecional crítica } \\
\text { velocidade orbital limite (1); }\end{array}$ & fundo; & & & \\
\hline
\end{tabular}


componente resultante do fluxo na estimativa do parâmetro crítico limite de cisalhamento. Correntes de maré, correntes geradas por ventos e correntes de densidade, em geral atuam simultaneamente sobre o fundo. 0 escoamento próximo ao fundo apresenta, por isso, diferentes componentes combinados e tanto a resultante do fluxo quanto seu estresse cisalhante deve ser 0 resultado destes efeitos combinados (LARSEN et al., 1981).

\section{Ação de correntes de fundo}

ZARILLO (1982) através de regressão linear múltipla observou que a energia do escoamento, a profundidade, o diâmetro médio dos grãos e a velocidade de cisalhamento são fortemente relacionadas à altura das formas de fundo. A associação desses parâmetros na forma de diagramas mostra boa aplicabilidade na estimativa de velocidades associadas às estruturas. Dessas estimativas, observou-se uma gradação na magnitude das correntes a $1,0 \mathrm{~m}$ do fundo $\left(U_{100}\right)$ variando entre $46 \mathrm{~cm} / \mathrm{s}$ (areias médias) e $34 \mathrm{~cm} / \mathrm{s}$ (areias muito finas) de leste para oeste. Esse comportamento está de acordo com a variação do índice RI. A velocidade média do fluxo estacionário (U) variou de forma diferente nesta área com uma gradação inversa em relação a $U_{100^{\prime}}$ sendo da ordem de $54 \mathrm{~cm} / \mathrm{s}$ a leste no local 6 e de $64 \mathrm{~cm} / \mathrm{s}$ a oeste no local 12 . Essas diferenças entre as duas estimativas, pode ser um indício de que outros agentes (ondas por exemplo), além de correntes de fundo, estejam contribuindo na geração das estruturas sedimentares e no selecionamento médio dos grãos.

\section{Ação de ondas}

Comparando as velocidades orbitais estimadas (Um1 e Um2), observa-se que as ondas têm condições de agir sobre o fundo do canal. Para quatro casos (Hs x T) de ondas observadas na região (Tabelas 3A e 3B), observou-se que: a) no caso 1 (média das alturas significativas máximas de verão), em todos os locais de estudo (exceto na área 17), estas ondas poderiam contribuir na geração das estruturas observadas. Nos locais onde as ações de ondas parecem exercer maior influência (11 e 12), as estruturas presentes poderiam estar vinculadas a eventos pretéritos de magnitudes menores, que estariam em equilíbrio no meio permanecendo preservadas, estando sujeitas, ainda, a um possível retrabalhamento por correntes de fundo tendo suas calhas parcialmente cobertas por sedimentos mais finos; b) no caso 2 (média das alturas significativas máximas anuais), haveria possibilidade de as ondas contribuírem na gênese das estruturas em qualquer dos locais de estudo, podendo ser uma boa evidência de que essas estruturas seriam ativas, e que estariam também sujeitas a modificações em sua geometria ao longo do ano. 0 fundo nesta região, portanto, seria móvel durante estes eventos (cerca de 12\% das observações) com velocidades orbitais da ordem de $50 \mathrm{~cm} / \mathrm{s}$; (c) nos casos 3 (média das alturas significativas

Tabela $3 a$ - Parâmetros para quatro situações de atividade de ondas na região do canal central da baía da llha Grande, na região de Itaorna, obtidos por Ferreira da Silva et al. (1996).

Table 3a - Four different wave conditions in the Ilha Grande Bay central channel. Data obtained by Ferreira da Silva et al. (1996) on the Itaorna region.

\begin{tabular}{ccccc}
\hline Parâmetros & Caso l & Caso 2 & Caso 3 & Caso 4 \\
\hline $\mathrm{Hs}(\mathrm{m})$ & 1.3 & 1.8 & 2.42 & 3.0 \\
$\mathrm{~T}(\mathrm{~s})$ & 9.7 & 10.3 & 11.9 & 10 \\
\hline
\end{tabular}

Tabela $3 b$ - Valores máximos de $U_{\mathrm{m} 2}$ em quatro casos de ondas capazes de agir sobre o fundo no local onde as formas de fundo foram observadas. Table $3 b-$ Maxima $_{U_{m 2}}^{U_{2}}$ velocities for seabed reworking conditions by waves in areas of bedform observed occurrences.

\begin{tabular}{ccccccc}
\hline Localização & $\begin{array}{c}U_{\mathrm{ml}} \\
\left(\mathrm{cm} \cdot \mathrm{s}^{-1}\right)\end{array}$ & $\begin{array}{c}\text { profundidade } \\
(\mathrm{m})\end{array}$ & $\begin{array}{c}\text { caso 1 } \\
\left(\mathrm{cm} . \mathrm{s}^{-1}\right)\end{array}$ & $\begin{array}{c}\text { caso 2 } \\
\left(\mathrm{cm}^{-1}\right)\end{array}$ & $\begin{array}{c}\text { caso 3 } \\
\left(\mathrm{cm} . \mathrm{s}^{-1}\right)\end{array}$ & $\begin{array}{c}\text { caso 4 } \\
\left(\mathrm{cm}^{-1} \mathrm{~s}^{-1}\right)\end{array}$ \\
\hline 6 & 28.0 & 16.0 & 39.13 & 56.06 & 80.12 & 91.85 \\
8 & 18.0 & 16.0 & 39.13 & 56.06 & 80.12 & 91.85 \\
9 & 18.0 & 16.3 & 38.52 & 55.19 & 79.36 & 90.53 \\
10 & 18.0 & 16.3 & 38.52 & 55.19 & 79.36 & 90.53 \\
11 & 18.0 & 16.5 & 38.10 & 54.79 & 78.63 & 89.84 \\
12 & 16.0 & 16.5 & 38.10 & 54.79 & 78.63 & 89.84 \\
17 & 35.0 & 21.0 & 30.95 & 44.89 & 66.02 & 73.23 \\
\hline
\end{tabular}


máximas de inverno) e 4 (condições esporádicas), todos os locais seriam fortemente afetados por velocidades orbitais da ordem de $80 \mathrm{~cm} / \mathrm{s}$ nesses eventos de mais alta energia, implicando em maior modificação nas estruturas sedimentares, bem como em maior disponibilidade de sedimentos para 0 transporte próximo ao fundo pelas correntes. É importante frisar que estas ondas foram observadas na porç̃̃o oeste do canal central, região de Itaorna. É possível que na região de Conceição de Jacareí, na porção leste do canal, a ação de ondas seja ligeiramente diferente. Porém, ainda não existem observações de ondas disponíveis para esta porção do canal. Em breve, a Petrobras pretende instalar nessa região uma bóia oceanográfica com sistema de medição de dados meteooceanográficos, em tempo real. Dessa forma, uma importante contribuição será efetuada na observação desses parâmetros, colaborando para a segurança das operações na baía.

\section{Formas de fundo e ecofácies}

Vários estudos vêm tentando distinguir as formas de fundo $\mathrm{e}$ entender como são geradas. Seus resultados ressaltam a dificuldade em se estabelecer normas gerais de investigação dessas estruturas, pois envolvem processos sujeitos a interações de variáveis sem comportamento linear em diferentes condições de fundo e de fluido (MILLER et al., 1977; LARSEN et al., 1981). A despeito das ressalvas feitas, as evidências observadas no presente estudo explicariam em parte a distribuição de ecofácies observada por Belo e outros, (em prep.), e dos contatos texturais sugeridos por Mahiques (1987) e Dias e outros (1990), nessa região do canal onde padrões de fundo lamosos (ecofácies VI e VII) adjacentes ao arenoso (ecofácies VIII), se intercalam com padrões intermediários (ecofácies III e IV) (Figura 4). Padrões estes, associados às formas de fundo observadas (Figura 8). Essa maneira de distribuição dos padrões de fundo, vinculada a oscilações de energia, é consistente a partir de observações feitas por MacCave (1971), embora no presente estudo não tenha sido enfocado o papel do material particulado em suspensão. Este autor sugere que a deposição de lama em geral é inibida pela ação de ondas e correntes de fundo com velocidades maiores que $40-50 \mathrm{~cm} /$ s. No entanto, quando há uma certa concentração de particulado em suspensão $(\sim 1,0 \mathrm{mg} / \mathrm{l})$ e baixa ação de ondas, a lama pode ser depositada por correntes de menor intensidade. Ou seja, podem existir áreas onde ocorre um balanço entre a concentração de partículas em suspensão e a atividade de ondas no controle deposicional. A simples existência de uma zona de alta atividade de ondas ou de fortes correntes de maré, por exemplo, não inibiriam a deposição lamosa quando a concentração de finos fosse grande $(100 \mathrm{mg} / \mathrm{l})$. Porém, na região, essas informações ainda não existem. Larsen e outros. (1981) observaram que em fundos de padrões finos, correntes oscilatórias geradas por ondas causam ressuspensão desses sedimentos que são advectados junto ao fundo e que podem preencher depressões no leito marinho, tais como as calhas de estruturas sedimentares.

As características das estruturas sedimentares de fundo e as estimativas de velocidades, interpretadas à luz de características do fundo marinho da baía (topografia submarina heterogênea e coluna d'água estratificada), sugerem, portanto, que haja na região centro-leste do canal central uma ação principal de correntes de fundo de sentido provavelmente SSE-NNW, associada a células de circulação do canal. No local 6, associada a um padrão de fundo grosso (ecofácies VIII), a direção predominante de correntes teria sentido NE-SW, e seria mais importante em relação à ação de ondas. Nos locais 8,9 e 10, associados a um tipo transitório (ecofácies III), ocorreriam açōes combinadas, provavelmente transversas, devido à variaçãa de orientação das estruturas. E nos locais 11 e 12, associados aos padrões mais finos (ecofácies VI e VII), haveria uma ação predominante de ondas de SE em relação a ação de correntes. Esses processos se refletiriam no grau de selecionamento dos sedimentos de fundo e na geração de formas de fundo na baía da llha Grande.

\section{CONCLUSÃO}

A partir de parâmetros obtidos das formas de fundo observadas em registros de sonar de varredural lateral, e com base em diagramas relacionando o diâmetro médio dos grãos, a profundidade e 0 tipo de estrutura presente, foram estimadas as velocidades de fluxos associadas à ação de correntes de fundo e à ação de ondas. No caso de correntes de fundo, os valores são parecidos com dados medidos no canal. Estes variam de $46 \mathrm{~cm} / \mathrm{s}$ (areias médias) a $34 \mathrm{~cm} / \mathrm{s}$ (areias muito finas) - de leste para oeste. 0 estudo de quatro casos de ação de ondas na região indicou que as ondas contribuem na gênese das estruturas sedimentares com velocidades orbitais entre 50 e $80 \mathrm{~cm} / \mathrm{s}$, sendo uma boa evidência de que estas seriam ativas e sujeitas a modificações em sua geometria ao longo do ano. Em eventos de mais alta energia, haveria maior disponibilidade de sedimentos para o transporte próximo a fundo pelas correntes. As características das estruturas sedimentares e as velocidades estimadas sugerem, portanto, que haja ações combinadas de ondas e de correntes no canal central. Os processos atuantes na parte central da baía da Ilha Grande seriam predominantemente de baixa energia e a sedimentação controlada por forçantes hidrodinâmicos, provavelmente com influência de forçantes atmosféricos. Estes processos definiriam a distribuição espacial dos padrões sedimentares de fundo na baía da llha Grande. 


\section{Agradecimentos}

Ao Programa de Recursos Humanos da ANP para o Setor Petróleo e Gás (PRH-ANP/MME/MCT), pelos recursos. Processo no: 48610.002734/ 99-01 / Matrícula no: 1999.0028-7;

Ao Laboratório de Geologia Marinha (LAGEMAR-UFF) pela infraestrutura, e ao PGGM, pelo uso dos equipamentos geofíisicos;

À SR2/UERJ e ao Dr. Marcelo Sperle (LOG-UERJ) por parte dos recursos para os levantamentos geofísicos de maio e dezembro de 1999 e ao Dr. Gilberto Dias (LAGEMAR-UFF), pela embarcação (ANITA-RIO);

À Dr. Isa Brehme (LAGEMAR-UFF), à Dr. Maria Augusta M. Silva (LAGEMAR-UfF), ao Dr. Cleverson G. Silva (LAGEMAR-UFF), a Dr. Michael Mahiques (IOUSP), a colega MSc. Eric Ribeiro (CENPESPetrobras) e ao revisor anônimo, pelas críticas e sugestôes ao manuscrito.

\section{REFERÊNCIAS}

ALLEN, J.R.L. Simple models for the shape and symetry of tidal sand waves: (1) statically stable equilibrium forms. Marine Geology, New York, v. 48, p. 31-49, 1982.

AMOS, C. L.; COLLINS, M. B. The combined effects of wave motion and tidal currents on the morfology of intertidal ripple marks: the Walsh, U.K. Journal of Sedimentary Petrology, [S.I.], v. 48, n. 3, p. 849-856, 1978.

CORRÊA, I. S. C. et al. Sedimentologia da parte central da Baía da Ilha Grande, Estado do Rio de Janeiro - Brasil. Notas Técnicas. (ECO/G/ UFRGS, [S.I.], n. 10, p. 61-71, 1997.

DIAS, G. T. M.; PEREIRA, M. de A. A.; DIAS, I. de M. Mapa geológico geomorfológico da Baía da Ilha Grande e Zona Costeira adjacente, esc. 1:80000. Universidade Federal Fluminense. Relatório Interno LAGEMAR, 1990.

Classificação de sedimentos marinhos inconsolidados: proposta de representação em cartas sedimentológicas. In: Congresso Brasileiro de Geologia, 39., 1996, Salvador. Anais.... Salvador: SBG, 1996.

FERREIRA DA SILVA, L. C. et al. Litoral Sul Fluminense: síntese do conhecimento das condições climatológicas e da dinâmica costeira. Universidade do Estado do Rio de Janeiro. Relatório Técnico para Furnas Centrais Elétricas. 1996.

FISH, J. P.; CARR, H. A. Sound Underwater Images: a guide to the generation and interpretation of side scan sonar data. $E G \& G$ Marine Instruments, Burlington, p. 11-15, 1990.

FRAGOSO, M. R. Estudo numérico da circulação marinha da região das Baías de Sepetiba e Il/ha Grande (RJ). 1999. Dissertação (Mestrado)-
Instituto Oceanográfico da Universidade de São Paulo, Universidade de São Paulo, São Paulo, 1999.

HENNINGS, I.; LURIN, B.; VERNEMMEN, C.; VANHESSCHE, U. On the behavior of tidal currents directions due to the presence of submarine sand waves. Marine Geology, New York, v. 69, p. 57-68, 2000.

IKEDA, Y.; STEVENSON, M.R. Determination of circulation and short period fluctuation at Itha Grande Bay (Rio de Janeiro-Brazil). Bolm Instituto Oceanográfico, São Paulo, v. 29, n. 1. p. 89-98,1980.

Seasonal characteristics of hidrography, turbulence and dispersion near Itha Grande (Rio de Janeiro-Brazil), based on RN Prof. W. Besnard. Bolm Instituto Oceanográfico, São Paulo, v. 31, n. 1, p. 11-32, 1982.

IKEDA, Y.; GODOI, S. S.; CACCIARI, P. L. Um estudo de séries temporais de corrente na Baía da llha Grande (Rio de Janeiro-Brasil). Bolm Instituto Oceanográfico, São Paulo, v. 1, n. 28, p. 1-24, 1989.

KOMAR, P. D.; MILLER, M. C. On the comparison between the thresold of sediment motion under waves and unidirectional currents with a discussion of the practical evaluation of the thresold. Journal of Sedimentary Petrology, [S.I.], v. 45, p. 362-367, 1975.

LARSEN, L. H. et al. Field investigations of the thresold of grain motion by ocean waves and currents. Marine Geology, New York, v. 42, p. 105$132,1981$.

LARSONNEUR, C.; BLOUYSSE, P.; AUFFRET, J. P. The surficial sediments of the english channel and its western approaches. Sedimentology, Oxford, v. 29, n. 6, p. 851-864, 1982.

LEE YOUNG, J.S.; SLEATH, J. F. A. Ripple formation in combined transdirectional steady and oscillatory flow. Sedimentology, Oxford, v. 37, p. 509-516, 1990.

MAHIQUES, M. M. Considerações sobre os sedimentos de superficie de fundo da Baía da Ilha Grande (Rio de Janeiro-Brasil). 1987. 2 v. Dissertação (Mestrado)-Instituto Oceanográfico da Universidade de São Paulo, Universidade de São Paulo, São Paulo, 1987.

MCCAVE, I.N. Wave effectiveness at the sea bed and its relationship to bedforms and deposition of mud. Journalof Sedimentary Petrology, [S.I.], v. 41, n. 1, p. 89-96, 1971.

MILLER, M. C.; MCCAVE, I. N.; KOMAR, P. D. Thresold of sediment motion under unidirectional currents. Sedimentology, [S.I.], v. 24, p. 507-527, 1977.

MIRANDA, L.B. Note on the occurrence of saline fronts in the llha Grande (Rio de Janeiro-Brazil). Bolm. Instituto Oceanográfico, São Paulo, v. 26, n. 2, p. 249-256, 1977.

REINECK, H. E; SINGH, I. B. Depositiona/ Sedimentary Environments. New York: Springer, 1975. 
RUBIN, D.M.; MCCULLOCH, D. S. The movement and equilibrium of bedforms in central San Francisco Bay. Menlo: U.S. Geological Survey, 1979. p. $97-113$.

SHEPARD, F. P.; MOORE, D. G. Sedimentary environments differentiated by coarse-fraction studies. Bull. Amer. Assoc. Petrol. Geologists, [S.I.], v. 38, p. 1772-1802, 1954.

SIGNORINI, S. R. A study of circulation in Bay of Ilha Grande and Bay of Sepetiba. Bolm. Instituto Oceanográfico, São Paulo, v. 29, n. 1, p. 41$55,1980$.

STERNBERG, R. W. Mesurements of incipient motion of sediment particles in the marine environment. Marine Geology, New York, v. 10, p. 113$119,1971$.
SOUTHARD, J. B. Representation of bed configurations in depth-velocitysize diagrams. Journal of Sedimentary Petrology, [S.I.], v. 41, n. 4, p. 903-915, 1971.

Depositional environments as interpreted from primary structures and stratification sequences. Dallas: Sepm, 1975.

SUGUIO, K. Introdução à sedimentologia. São Paulo: Edgar Blucher, 1973.

WIEGEL, R. L. Gravity waves: tables of functions. Berkeley: Council on Wave Research, The Engineering Foundation, 1954.

ZARILLO, G. A. Stability of bedforms in a tidal environment. Marine Geology, New York, v. 48,. p. 337-351, 1982.

\section{NOTA SOBRE O AUTOR}

Wellington Ceccopieri Belo é formado em Eletrônica (CEFET-RJ/1988), Graduado em Oceanografia (UERJ/1998), e Mestre em Geologia/Geofísica Marinha (LAGEMAR-UFF-ANP/2001). Tem atuado nas áreas de Oceanografia Geológica e Meteo-Oceanografia. Participou no desenvolvimento de um sistema de monitoramento em tempo real de parâmetros meteo-oceanográficos, junto à CHEMTECH/SIEMENS, para a PETROBRAS. É Oceanógrafo do Centro de Pesquisas da PETROBRAS (CENPES), onde colabora na investigação do clima de vórtices e meandros do Sistema Corrente do Brasil (CB/CCI), junto ao I0-USP, e no desenvolvimento de parâmetros de projeto para unidades de produção marinhas. Seus interesses de pesquisa envolvem dinâmica de sedimentos e instabilidades baroclínicas do escoamento da Corrente do Brasil. 